\title{
A NEW SYNTHESIS OF PHENYLIMIDAZO THIAZOLO 2,3- DIMETHYL BENZOCYCLOHEPTENE
}

\author{
Kakam Srinivas*, Manne Satyanarayana Reddy and Gudipati Srinivasulu \\ Research \& Development, Dr. Reddy's Laboratories Ltd, Bulk Actives Unit-Ill, \\ Bollaram, Jinnaram, Medak Dist 502 325, A. P. e. mail: katkam@drreddys.com
}

\begin{abstract}
New heterocyclic system namely 2,3-dimethyl-10-phenyl-6,7-dihydro-5H-benzo[6,7] cyclohepta[1,2-d]imidazo[2,1-b][1,3]thiazole 4 has been synthesized via the reaction of 2 -(2-imino-1,4,5,6tetrahydro-2H-benzo[7,8]cyclohepta[d][1,3]thiazol)-I-phenyl-I-ethene-1-ol 3 intermediate with phenacyl bromide, in good yield.
\end{abstract}

\section{Introduction}

A number of biologically interesting polynuclear compounds incorporating a fused thiophene ring viz. thiasteroids ${ }^{1}$, analogues of indole alkaloids ${ }^{2,3}$, carcinogenic compounds ${ }^{4}$ etc., consists of sixmembered ring annelated to thiophene. But examples of polycondensed systems incorporating a thiophene, imidazole or thiazole ring fused to seven membered ring (viz benzosuberones and benzazepines) are sparse. In continuation of previous studies ${ }^{3-7}$ in the synthesis of biologically active fused heterocycles we have synthesized the hitherto unreported 2,3-dimethyl phenylimidazo thiazolobenzocyclo heptene derivative 4 starting from the 2,3-dimethyl-6,7,8,9tetrahydro-5H-benzocyclo hepten-5-one $\mathbf{1}^{8}$.

\section{Chemistry}

Reaction of the 2,3-dimethyl-6,7,8,9,-tetrahydro-5H-benzocyclohepten-5-one 1 with thiourea, MCM-41 $(\mathrm{H})$ and iodine ${ }^{9}$ were heated under reflux to give corresponding 5,6-dihydro-4Hbenzo[3,4]cyclohepta[d][1,3]thiazol-2-amine 2 as colourless crystals $(58 \%)$. Cyclization of 2 with phenacyl bromide at room temperature resulted in dimethyl 2-(2-imino-1,4,5,6-tetrahydro- $2 \mathrm{H}$ benzo[7,8]cyclohepta[d][1,3]thiazol-1-yl)-1-phenyl-1-ehene-1-ol 3 as a intermediate product. Subsequently compound 3 was assigned the enol form and gave 2,3-dimethyl-10-phenyl-6,7dihydro-5H-benzo[6,7]cyclohepta

[1,2-d]imidazo[2,1-b] [1,3] thiazole 4 heating in ethanol Scheme-I. Their structures were established by 'H NMR, Mass, IR and elemental analysis. 

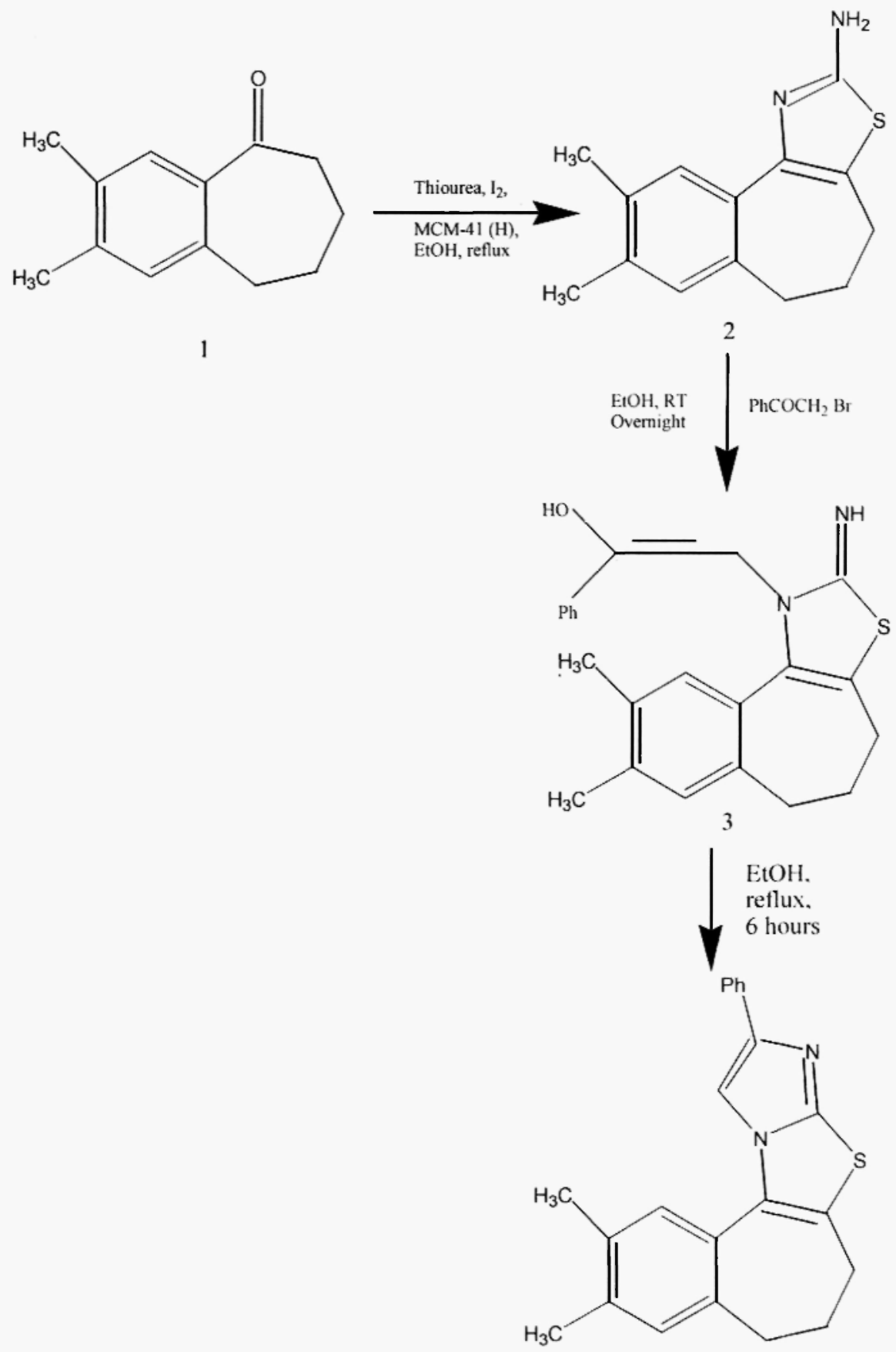

4

\section{Scheme-I}




\section{Experimental}

Melting points were determined in open glass capillaries on a polmon melting point apparatus and are uncorrected. 'H NMR spectra were recorded on a Gemini (200MHZ) spectrometers (Chemical shifts are recorded in $\delta, \mathrm{ppm}$ ); internal standard was TMS and IR spectra were recorded in $\mathrm{KBr}$ on a Perkin-Elmer bio- spectrometer. Elemental analyses were carried out with Carlo-Ebra 1106 Elemental Analyzer.

8, 9 - Dimethyl $-5,6$ dihydro - $4 \mathrm{H}$ - benzo $[3,4]$ cyclohepta $[\mathrm{d}][1,3]$ thiazol - 2 amine (2):

A mixture of $1(20 \mathrm{mmol})$, thiourea $(7 \mathrm{mmol})$ iodine $(20 \mathrm{mmole})$ and $\mathrm{MCM}-41(\mathrm{H})$ a pinch were refluxed for $52 \mathrm{hrs}$, in absolute Ethanol $(60 \mathrm{ml})$. At this point TLC showed only a slight change in the substrate. After prolonged refluxing (6 days until TLC showed the absence of the Ketone) the resulting hydride was dissolved in hot water. The solution was filtered while hot and the clear filtrate was neutralized with a strong solution of ammonia. The resulting precipitate was washed with water and crystallized from ethanol.

Yield $56 \%$, colourless crystalline powder, m.p., $278.6^{\circ} \mathrm{C}$; IR $(\mathrm{KBr}): \gamma 3365 \mathrm{~cm}^{-1}$; ${ }^{\prime} \mathrm{H}$ NMR $\left(\mathrm{DMSO}-\mathrm{d}_{6}\right): \delta 2.10-2.42(\mathrm{~m}, 2 \mathrm{H}, 5-\mathrm{H}), 2.65-2.88(\mathrm{~m}, 4 \mathrm{H}, 4 \& 6-\mathrm{H}), 5.63(\mathrm{br}, 2 \mathrm{H}$, $\mathrm{NH}_{2}, \mathrm{D}_{2} \mathrm{O}$ exchangeable), $2.25\left(\mathrm{~s}, 6 \mathrm{H}, 2 \mathrm{CH}_{3}\right), 6.52(\mathrm{~s}, 1 \mathrm{H}, 7-\mathrm{H})$ and $7.31(\mathrm{~s}, 1 \mathrm{H}, 10-\mathrm{H})$. Anal. Caled for : $\mathrm{C}_{14} \mathrm{H}_{16} \mathrm{~N}_{2} \mathrm{~S}$ : C, 64.53; H,6.19; N, 10.75\%, Found : C, $64.55 ; \mathrm{H}, 6.08 ; \mathrm{N}, 10.77 \%$.

2 - (2 - Imino - 8, 9 - dimethyl -1, 4, 5, 6 - tetrahydro - 2 H - benzo [7, 8] cyclohepta $[\mathrm{d}][1,3]$ thiazol - $1-$ yl) - 1 - phenyl - 1 - ethene - 1 - ol (3) :

A mixture of $2(16 \mathrm{mmol})$ and phenacyl bromide $(16 \mathrm{mmol})$ in $60 \mathrm{ml}$ ethanol were allowed to stand at room temperature overnight. The crystals, which separated, were collected by filtration and washed with a $10 \mathrm{ml}$ of ethanol.

Yield $72 \%$, m.p. $236^{\circ} \mathrm{C} ; \quad$ IR $(\mathrm{KBr}) ; \gamma 3366,2890,2865 \mathrm{~cm}^{-1}$.

${ }^{1} \mathrm{H}$ NMR (DMSO - $\left.\mathrm{d}_{6}\right): \delta 2.19-2.35(\mathrm{~m}, 2 \mathrm{H}, 5-\mathrm{H}), 2.68-2.80(\mathrm{~m}, 4 \mathrm{H}, 4 \& 6-\mathrm{H}), 7.16(\mathrm{~S}$, $1 \mathrm{H},=\mathrm{CH}), 8.80(\mathrm{brs}, 1 \mathrm{H},=\mathrm{NH}), 8.78(\mathrm{~s}, 1 \mathrm{H},-\mathrm{OH}), 2.26\left(\mathrm{~s}, 6 \mathrm{H}, 2 \mathrm{CH}_{3}\right), 6.48(\mathrm{~s}, 1 \mathrm{H}, 7-\mathrm{H})$, $7.25(\mathrm{~s}, 1 \mathrm{H}, 10-\mathrm{H}), 6.28-7.25(\mathrm{~m}, 5 \mathrm{H}, \mathrm{Ar}-\mathrm{H})$.

Anal. Caled. For $\mathrm{C}_{22} \mathrm{H}_{22} \mathrm{~N}_{2} \mathrm{OS} ： C, 72.89 ; \mathrm{H}, 6.11 ; \mathrm{N}, 7.72 \%$,

$$
\text { Found : } \quad \mathrm{C}, 72.83 ; \mathrm{H}, 6.09 ; \mathrm{N}, 7.77 \%
$$


2, 3 - Dimethyl - 10 - phenyl - 6, 7 - dihydro -5 H - benzo [6, 7] cyclohepta $[1,2$ d] imidazo $[2,1-b][1,3]$ thiazole $(4)$ :

A suspension of $3(15 \mathrm{mmol})$ in $50 \mathrm{ml}$ ethanol was heated under reflux 6.5 hours after cooling, the crystals which separated were collected by filtration.

Yield $68 \%$, buff white powder, m.p. $>290^{\circ} \mathrm{C}(\mathrm{dec}) ;{ }^{1} \mathrm{H}$ NMR $\left(\right.$ DMSO $\left.-\mathrm{d}_{6}\right): \delta 2.18-2.35$ $(\mathrm{m}, 2 \mathrm{H}, 6-\mathrm{H}), 2.65-2.88(\mathrm{~m}, 4 \mathrm{H}, 5 \& 7-\mathrm{H}), 7.55(\mathrm{~s}, 1 \mathrm{H}, 11-\mathrm{H}), 2.26\left(\mathrm{~s}, 6 \mathrm{H}, 2 \mathrm{CH}_{3}\right), 7.25$ $(\mathrm{s}, 1 \mathrm{H}, 1-\mathrm{H})$ and $6.31-7.45(\mathrm{~m}, 5 \mathrm{H}, \mathrm{Ar}-\mathrm{H})$ MS: $\mathrm{m} / \mathrm{z} 344\left(\mathrm{M}^{+}\right), 329,267,244,199(100 \%), 171,131,91,77,64$.

Anal., caled for $\mathrm{C}_{22} \mathrm{H}_{20} \mathrm{~N}_{2} \mathrm{~S}$ : C, $76.70 ; \mathrm{H}, 5.85 ; \mathrm{N}, 8.13 \%$.

Found : $\mathrm{C}, 76.70 ; \mathrm{H}, 5.88 ; \mathrm{N}, 8.21 \%$

\section{References}

1. S.R. Ramdas, P.C. Chennaiah, N.S. Chandra Kumar, M.V. Krishna, P.S. Srinivasan, Sastry and S. Apparao, Heterocycles, 19, 861 (1982).

2. T.R. Bosin and E. Campaigne, Adv., Drug Res., 12, 191 (1977).

3. E. Campaigne, D.R. Knapp, E.S. Neiss and T.R.Bosin, Adv. Drug Res., 5 (1970).

4. B.D. Tilak, Tetrahedron, 9, 76 (1960).

5. J. McLean, V. Peesapati and G.R. Proctor, J. Chem. Soc., Perkin Trans 1, 98 (1979).

6. N. Lingaiah and V. Peesapati, Org. Prep. Proced. Int., 24, 27 (1992).

7. N. Lingaiah, and V. Peesapati, Org., Prep. Proced. Int., 25, 5 (1993).

8. G. R. Proctor, J. Chem. Soc., 4274 (1964).

9. R.M., Dodson and L.C. King, J. Am, Chem. Soc., 67, 2242 (1945).

\section{Received on October 8, 2003.}

